\title{
Altered White Matter Microstructure in Adolescents and Adults with Bulimia Nervosa
}

\author{
Xiaofu He', Mihaela Stefan', Kate Terranova', Joanna Steinglass² and Rachel Marsh ${ }^{*, 1,2}$ \\ 'Division of Child and Adolescent Psychiatry, Department of Psychiatry, New York State Psychiatric Institute and College of Physicians \& Surgeons, \\ Columbia University, New York, NY, USA; ${ }^{2}$ Eating Disorders Research Unit, Department of Psychiatry, New York State Psychiatric Institute and \\ College of Physicians \& Surgeons, Columbia University, New York, NY, USA
}

\begin{abstract}
Previous data suggest structural and functional deficits in frontal control circuits in adolescents and adults with bulimia nervosa (BN), but less is known about the microstructure of white matter in these circuits early in the course of the disorder. Diffusion tensor imaging (DTI) data were acquired from 28 female adolescents and adults with BN and 28 age- and BMI-matched healthy female participants. Tract-based spatial statistics (TBSS) was used to detect group differences in white matter microstructure and explore the differential effects of age on white matter microstructure across groups. Significant reductions in fractional anisotropy (FA) were detected in the BN compared with healthy control group in multiple tracts including forceps minor and major, superior longitudinal, inferior fronto-occipital, and uncinate fasciculi, anterior thalamic radiation, cingulum, and corticospinal tract. FA reductions in forceps and frontotemporal tracts correlated inversely with symptom severity and Stroop interference in the BN group. These findings suggest that white matter microstructure is abnormal in $\mathrm{BN}$ in tracts extending through frontal and temporoparietal cortices, especially in those with the most severe symptoms. Age-related differences in both FA and RD in these tracts in BN compared with healthy individuals may represent an abnormal trajectory of white matter development that contributes to the persistence of functional impairments in self-regulation in BN.

Neuropsychopharmacology (2016) 4I, I84I-1848; doi:I0.1038/npp.20I5.354; published online 6 January 2016
\end{abstract}

\section{INTRODUCTION}

Bulimia nervosa $(\mathrm{BN})$ is defined by the presence of recurrent binge-eating episodes that are accompanied by a sense of loss of control and followed by self-induced vomiting or another compensatory behavior to avoid weight gain. BN primarily affects females and typically begins during adolescence, a period of brain maturation during which structural and functional brain changes occur that may relate to adolescentspecific behaviors and characteristics such as risk taking and impulsivity (Romer, 2010), as well as the adolescent emergence of psychiatric illnesses (Giedd and Rapoport, 2010). In healthy individuals, white matter (WM) microstructure continues to change over adolescence and into adulthood (Lebel and Beaulieu, 2011; Lebel et al, 2008), specifically in tracts connecting the frontal cortex with other cortical and subcortical regions (Asato et al, 2010), with frontotemporal connections demonstrating the most prolonged development. Thus, uncovering WM abnormalities in individuals with $\mathrm{BN}$ may help to explain its typical emergence during adolescence and persistence into adulthood.

*Correspondence: Dr R Marsh, Division of Child and Adolescent Psychiatry in the Department of Psychiatry, Columbia University and New York State Psychiatric Institute, 105I Riverside Drive, Unit 74, New York, NY I0032, USA, Tel: + I 646774 5774, Fax: + I 212543 0522, E-mail: MarshR@nyspi.columbia.edu

Received 7 April 2015; revised 12 November 2015; accepted 4 December 2015; accepted article preview online 9 December 2015
Our previous functional neuroimaging findings from adult and adolescent females with $\mathrm{BN}$ suggest that a failure to engage frontal regions may contribute to an impaired capacity for self-regulation (Marsh et al, 2009), early in the course of the illness (Marsh et al, 2011). Our previous structural MRI data revealed that local volumes of bilateral frontal and temporoparietal cortices were reduced in $\mathrm{BN}$ compared with healthy participants (Marsh et al, 2015). These data also suggested that reductions in inferior frontal cortices are associated with, and may underlie, these regulatory deficits in individuals with $\mathrm{BN}$, likely contributing to their impaired control over eating and other behaviors (Marsh et al, 2015). Because these reductions became more prominent with age in $\mathrm{BN}$, we suggested that they might represent an abnormal developmental trajectory such as accelerated gray matter loss, axonal pruning, or reduced myelination in frontal cortices. Data from the only extant diffusion tensor imaging (DTI) study of individuals with BN suggest reduced WM integrity in adult patients compared with healthy women in bilateral corona radiata extending to the posterior limb of the internal capsule, corpus callosum, right subinsular WM, and right fornix (Mettler et al, 2013). These data were interpreted in relation to altered taste, body perception, and reward processing in BN. Unknown; however, is whether WM microstructure is abnormal earlier in the course of the disorder or whether such abnormalities might contribute to self-regulatory deficits in $\mathrm{BN}$. 
Thus, the objective of this study was to assess WM microstructure in adolescents and adults with BN compared with age-matched healthy participants. Based on previous DTI findings (Mettler et al, 2013) and our previous findings from women with $\mathrm{BN}$, we hypothesized that compared with control participants, those with BN would have reduced fractional anisotropy (FA) in multiple WM tracts, specifically those extending to and from frontal and temporoparietal areas. FA describes the distribution of water diffusion within a voxel and is thought to reflect properties of WM indices that can also be derived to add more information about WM architecture, such as mean, axial, and radial diffusivity (ie, $\mathrm{MD}, \mathrm{AD}$, and $\mathrm{RD}$, respectively). To extend our previous study of surface morphology in these same individuals (Marsh et al, 2015), we also explored group differences in the age correlates of FA and whether abnormalities in the WM microstructure of individuals with BN were associated with measures of BN symptom severity or with deficits in self-regulatory control, as measured by cognitive interference on a Stroop task (Stroop, 1935) performed outside of the MRI scanner.

\section{MATERIALS AND METHODS}

\section{Participants}

Diffusion data were acquired from 35 adolescent and adult females with $\mathrm{BN}$ and 37 age- and BMI-matched healthy control (HC) females. A total of 33 of the BN and 28 of the HC participants had participated in our previous structural (Marsh et al, 2015) and functional (Marsh et al, 2009, 2011) MRI studies. Those with BN were recruited through flyers posted in the local community and advertisements posted on Internet sites (such as craigslist.com and eating disorderspecific websites) and through the New York State Psychiatric Institute (NYSPI) Eating Disorders Clinic where some were receiving treatment. Control participants were recruited through flyers and Internet advertisements. Those with a history of neurological illness, past seizures, head trauma with loss of consciousness, mental retardation, pervasive developmental disorder, or current Axis I disorders (other than major depression for the BN participants) were excluded. Controls also had no lifetime Axis I disorders. Formal diagnoses of BN and comorbid neuropsychiatric diagnoses were established using standard adult and child measures (Supplementary Material). All participants received monetary compensation for their participation. The institutional review board of the NYSPI approved this study, and all participants gave informed consent.

\section{DTI Data Acquisition}

MRI data were acquired on a GE Signa 3.0 Tesla whole body scanner (GE Medical Systems, Waukesha, Wisconsin). Diffusion data were measured along 25 noncollinear spatial directions using a single-shot spin echo planar imaging sequence $(\mathrm{TR} / \mathrm{TE}=17000 \mathrm{~ms} / 90 \mathrm{~ms}$, flip angle $=90$, field of view $=24 \mathrm{~cm}$, matrix size $=132 \times 128$ (machine-interpolated to $256 \times 256$ for postprocessing), $\mathrm{b}$ value $=1000 \mathrm{~s} / \mathrm{mm}^{2}$, and three baseline images at $b=0 \mathrm{~s} / \mathrm{mm}^{2}$, slice thickness $=2.5 \mathrm{~mm}$ without gap, and an in-plane resolution of $0.94 \mathrm{~mm}$ ). Scan time for each excitation was $7 \mathrm{~min}$ and $56 \mathrm{~s}$ and we averaged 2 separate excitations $(\mathrm{NEX}=2)$ for each participant.

\section{DTI Data Processing}

DTI data were processed using the FMRIB Software Library (FSL) 5.0.6 (http://fsl.fmrib.ox.ac.uk/fsl/fslwiki/) (Smith et al, 2004). FMRIB diffusion toolbox (FDT) was used to assess $\mathrm{FA}, \mathrm{MD}, \mathrm{AD}$, and $\mathrm{RD}$. First, eddy currents that induce stretches and shears in the diffusion weighted images (DWIs) were corrected using FDT toolbox, and then brain was extracted using the Brain Extraction Tool (Smith, 2002) in order to delete nonbrain tissue from an image of the whole head. Second, a diffusion tensor model was fitted at each voxel using DTIFIT and $\mathrm{FA}, \mathrm{MD}, \mathrm{AD}$, and $\mathrm{RD}$ images were derived from the fitted diffusion tensors. Data quality was assessed by visually checking the raw DW images, eddy current corrected DW images, and the color-encoded FA images (He et al, 2014) to exclude participants who had motion-corrupted DTI data.

Voxelwise statistical analysis of the FA images was then carried out using tract-based spatial statistics (TBSS) (Smith et al, 2006). First, all subjects' FA images were aligned to a $1 \times 1 \times 1 \mathrm{~mm}$ standard space by running the nonlinear registration. The FMRIB58_FA standard-space image was used as the target image for registration (Smith et al, 2006) and the aligned FA images from each participant were then averaged to create a mean FA image. A mean FA skeleton image was generated by using a thinned group mean FA images, representing the centers of all WM tracts common to the group. The FA threshold for the skeleton was 0.20 to exclude gray matter regions from the analyses. Each participant's aligned FA images were then projected onto the skeleton that was then used for statistical analyses. Nonlinear warps and skeleton projection were then also applied to $\mathrm{MD}, \mathrm{AD}$, and $\mathrm{RD}$ images for statistical analyses.

\section{Stroop Interference}

Stroop interference, measured outside the scanner, was used for correlation analyses with FA because the resolution of interference on this task requires the engagement of selfregulatory control (Marsh et al, 2006). This task version consisted of three subtasks on which participants were asked to name the color of dots (task A), read words printed in black ink (task B), and name the color of printed words as quickly as possible (task C). The time to completion of each task was recorded and Stroop interference was calculated (Supplementary Material).

\section{Statistical Analyses}

To assess potential alterations in WM microstructure, permutation-based nonparametric inference $(n=10000)$ was used to perform statistical analyses on FA as well as $\mathrm{MD}, \mathrm{AD}$, and RD using FSL Randomize version 2.9 (Nichols and Holmes, 2002). Our a priori test of group differences in FA and our exploratory tests of group differences in MD, $\mathrm{AD}$, and $\mathrm{RD}$ were corrected for multiple comparisons with threshold-free cluster enhancement (TFCE, FWE-corrected $p<0.05)$ (Smith and Nichols, 2009). The Johns Hopkins University White-Matter Tractography Atlas (Oishi et al, 
Table I Demographic, Clinical, and Neuropsychological Characteristics of Participants

Participants

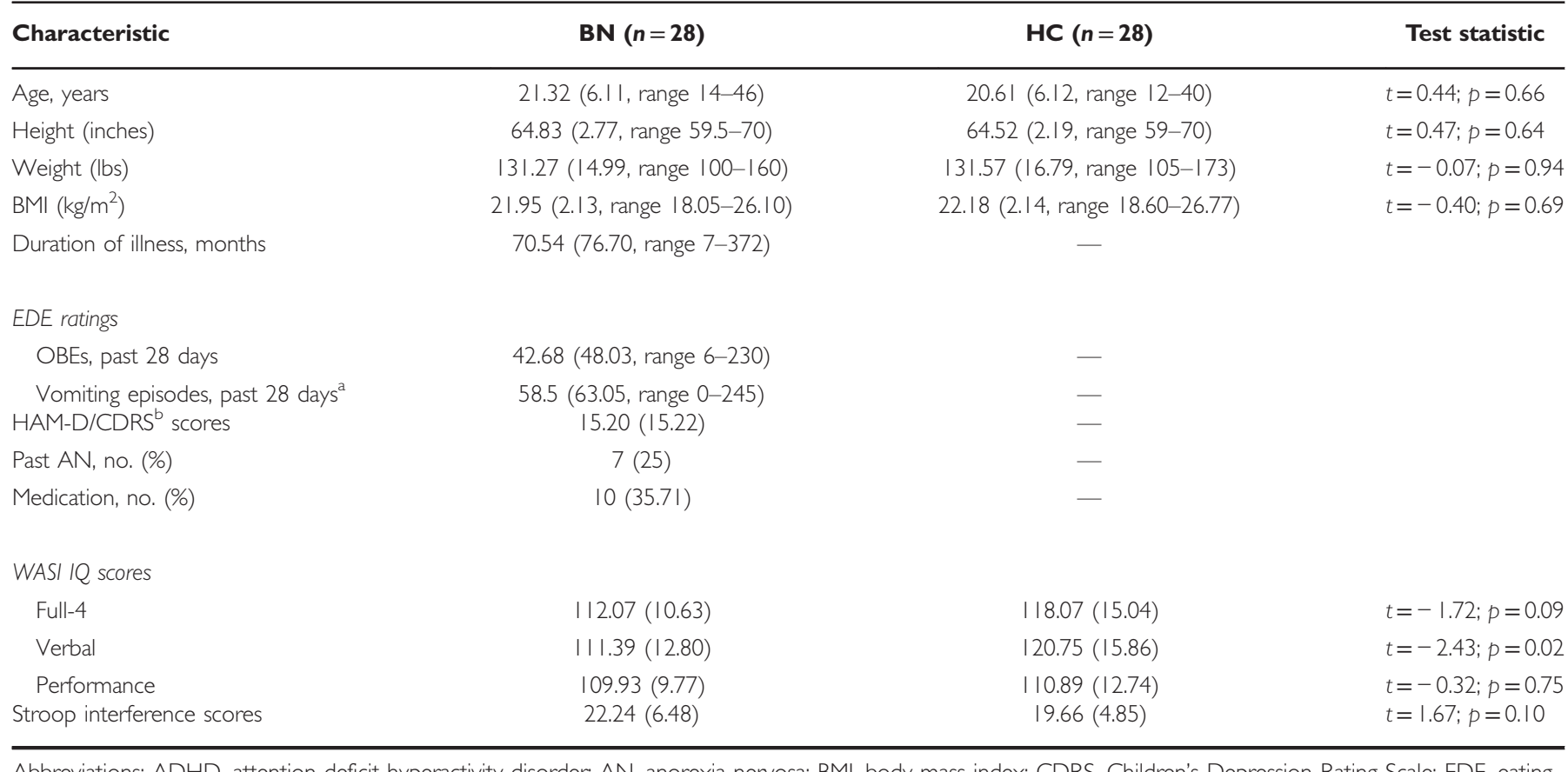

Abbreviations: ADHD, attention deficit hyperactivity disorder, AN, anorexia nervosa; BMI, body mass index; CDRS, Children's Depression Rating Scale; EDE, eating disorders examination; HAM-D, Hamilton Depression; OBE, objective bulimic episode; WASI, Weschler Abbreviated Scale of Intelligence.

Values are mean (SD) unless otherwise specified.

${ }^{a}$ Two adolescents in the Bulimia Nervosa group did not purge but engaged in driven exercise and abused laxatives in the past 28 days before participation.

${ }^{\mathrm{b}} \mathrm{CDRS}$ scores were collected in 4 adolescents with BN under 18 years of age, and HAM-D scores were collected in the other BN participants.

2011) was used to label WM tracts. Using linear regression models adjusting for age and/or illness duration $(p<0.05$, uncorrected), we also explored associations of FA with BN symptom severity in the $\mathrm{BN}$ group and compared the groups in their patterns of correlations of FA with age and Stroop interference in tracts where group differences were detected.

\section{RESULTS}

\section{Participants}

After removing the DTI data from $7 \mathrm{BN}$ and 9 control participants because of excessive motion, data from 28 female adolescents and adults with BN and 28 age-matched healthy females remained for analysis including $15 \mathrm{BN}$ and 15 healthy adolescents ( $\leqslant 19$ years). The majority ( $26 \mathrm{BN}$ and $25 \mathrm{HC}$ ) of these individuals also participated in our previous MRI studies (Marsh et al, 2009, 2011, 2015). All participants were right-handed. The BN group included 5 participants who were receiving inpatient treatment (scanned within 1 month of admission), 8 who were receiving outpatient psychotherapy, and 15 who were symptomatic but nontreatment seeking at the time of participation. None met criteria for major depressive disorder. Seven had a prior diagnosis of anorexia nervosa (AN). Ten were taking selective serotonin reuptake inhibitors (SSRIs) at the time of scanning. Table 1 shows additional demographic and clinical characteristics of both groups.

\section{A Priori Hypothesis Testing}

Compared with HC participants, those with $\mathrm{BN}$ had significantly smaller FA values in one large cluster (TFCE, FWE-corrected $p<0.05$ ) encompassing forceps minor and major, superior longitudinal fasciculus (SLF), SLF-temporal, inferior fronto-occipital fasciculus (IFO), anterior thalamic radiation (ATR), corticospinal tract (CST), uncinate fasciculus (UNC), and cingulum (cingulate gyrus) of both hemispheres (Figure 1 and Table 2). In no instance did participants with BN exhibit significantly more FA than $\mathrm{HC}$ participants.

\section{Exploratory Analyses}

Group differences in $R D$. Compared with HC participants, those with $\mathrm{BN}$ had larger $\mathrm{RD}$ values in many of the same tracts where FA values were smaller: forceps minor and major, left SLF, SLF-temporal, IFO, ATR, CST, and cingulum (cingulate gyrus) (TFCE, FWE-corrected $p<0.05$, Supplementary Figure S1). No significant group differences were detected in any tracts using $\mathrm{AD}$ and $\mathrm{MD}$.

Correlations with symptom severity. In the $\mathrm{BN}$ group, significant inverse associations of FA with log-transformed objective bulimic episodes (Walsh et al, 1997) were detected in forceps minor and major, left ATR, IFO, and SLF. Scatterplots of these associations revealed that the BN participants who engaged in most episodes in the 28 days before scanning had the least FA in these tracts 

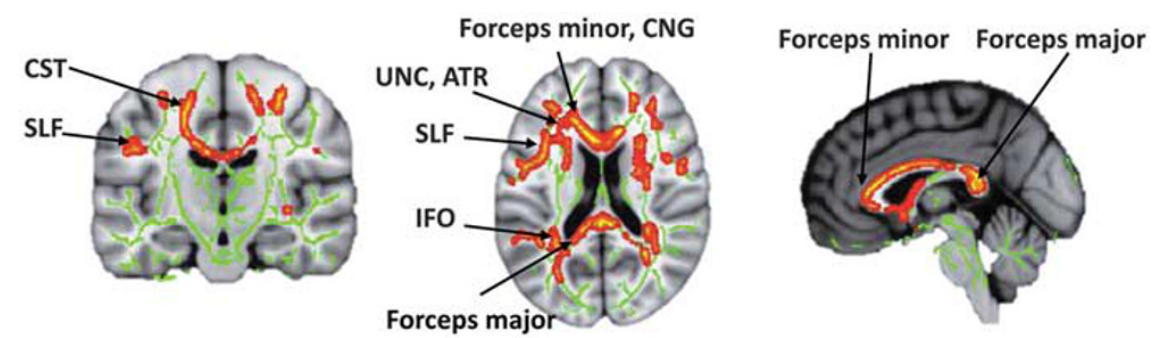

Figure I Group differences in white matter microstructure. Cluster in which less FA was detected in the BN compared with HC participants (thresholdfree cluster enhancement, FWE-corrected $p<0.05$ ). Regions showing smaller FA values are overlaid on the mean FA skeleton (green) and MNII52 TI map. The results are 'thickened' using tbss_fill script in FSL TBSS toolbox and displayed in neurological orientation for better visualization. ATR, anterior thalamic radiation; CNG, cingulum (cingulate gyrus); CST, corticospinal tract; IFO, inferior fronto-occipital fasciculus; SLF, superior longitudinal fasciculus; SLF-temporal, superior longitudinal fasciculus (temporal part); UNC, uncinate fasciculus.

Table 2 Cluster in Which FA Was Significantly Less in the BN Compared with HC Participants

\begin{tabular}{cccccc}
\hline Voxels & MIN p-value & MAX X (mm) & MAX Y (mm) & MAX Z (mm) & Major tracts $^{\mathbf{a}}$ \\
\hline 22280 & 0.024 & 5 & 4 & 24 & Forceps minor and major, SLF, SLF-temporal, IFO, ATR, CST, UNC, CNG
\end{tabular}

Abbreviations: ATR, anterior thalamic radiation; CNG, cingulum (cingulate gyrus); CST, corticospinal tract; IFO, inferior fronto-occipital fasciculus SLF, superior longitudinal fasciculus; SLF-temporal, superior longitudinal fasciculus (temporal part); UNC, uncinate fasciculus.

Voxels: the number of voxels in each cluster. MIN p-value: the value of the minimum p-value (threshold-free cluster enhancement, FWE-corrected $p$-value) within the cluster. MAX X/Y/Z (mm): the location of the voxel with minimum $p$-value in the standard space coordinates $(\mathrm{mm})$.

ajHU white-matter tractography atlas.

$(p<0.05$, Figure 2). Significant inverse associations of FA with log-transformed vomiting episodes were detected in the same tracts $(p<0.05$, Supplementary Figure S2) and scatterplots indicated the least FA in the BN participants who engaged in those most vomiting episodes. Finally, significant inverse associations of FA with ratings of preoccupation with shape and weight were also detected in forceps minor, left ATR, IFO, and SLF, with scatterplots suggesting the least FA in those who were the most preoccupied with their shape and weight $(p<0.05$, Supplementary Figure S3).

Age effects. Significant diagnosis-by-age interactions on FA were detected in forceps minor and major, bilateral ATR, CST, and IFO, and left SLF $(p<0.05$, Figure 3$)$. Scatterplots of these interactions indicated that FA in these tracts correlated positively with age in the $\mathrm{BN}$ group only. In addition, significant diagnosis-by-age interactions on $\mathrm{RD}$ were detected in forceps minor and major, left ATR, CST, and SLF $(p<0.05$, Supplementary Figure S4), with scatterplots indicating inverse correlations of $\mathrm{RD}$ with age in these tracts in the BN group. Removal of the oldest BN participant (46 years) revealed that these diagnosis-by-age interactions on FA and RD were not outlier driven $(p<0.05)$. In addition, these group differences in age effects remained when we controlled for illness duration in the BN sample (Supplementary Material and Supplementary Figure S5). Finally, analyses comparing FA and $\mathrm{RD}$ in the adolescent (15 BN, $15 \mathrm{HC}, \leqslant 19$ years) and adult (13 BN, $13 \mathrm{HC})$ groups separately revealed significant group differences in the adolescents only; compared with HC adolescents, those with BN had smaller FA and larger RD values in multiple tracts bilaterally, including forceps minor and major, ATR, SLF, SLF-temporal, IFO, inferior longitudinal fasciculus (ILF), CST, UNC, and cingulum (cingulate gyrus) (TFCE, FWE-corrected $p$ 's $<0.05$, Supplementary Figures S6a and $b$ ).

Interactions of diagnosis $\times$ Stroop interference. The BN and $\mathrm{HC}$ groups performed similarly on the Stroop task (Table 1). However, significant interactions of diagnosis-byStroop interference scores on FA were detected in forceps minor, left CST, SLF, and SLF-temporal, and right ATR and IFO $(p<0.05$, Supplementary Figure S7). FA in these tracts correlated inversely with Stroop scores in the BN and positively with Stroop scores in the healthy participants. Significant diagnosis-by-Stroop interactions on $\mathrm{RD}$ were also detected in left CST and IFO, as well as in forceps major $(p<0.05) . \mathrm{RD}$ in these tracts correlated positively with Stroop scores in the BN and inversely with Stroop scores in the healthy participants.

Medication and comorbidity effects. After including current SSRI treatment as an independent variable in a separate linear regression, significantly decreased FA values in the forceps minor and major, SLF, SLF-temporal, IFO, ATR, and CST in the BN group remained unchanged from those depicted in Figure 1. We additionally conducted a between-group analysis including only the $\mathrm{BN}$ participants who were not taking medications $(n=18)$. Both analyses indicated that SSRI treatment did not have an appreciable effect on our findings. Similarly, neither a history of anorexia nor current depressive symptoms contributed to our findings $(p ' s<0.05)$. 


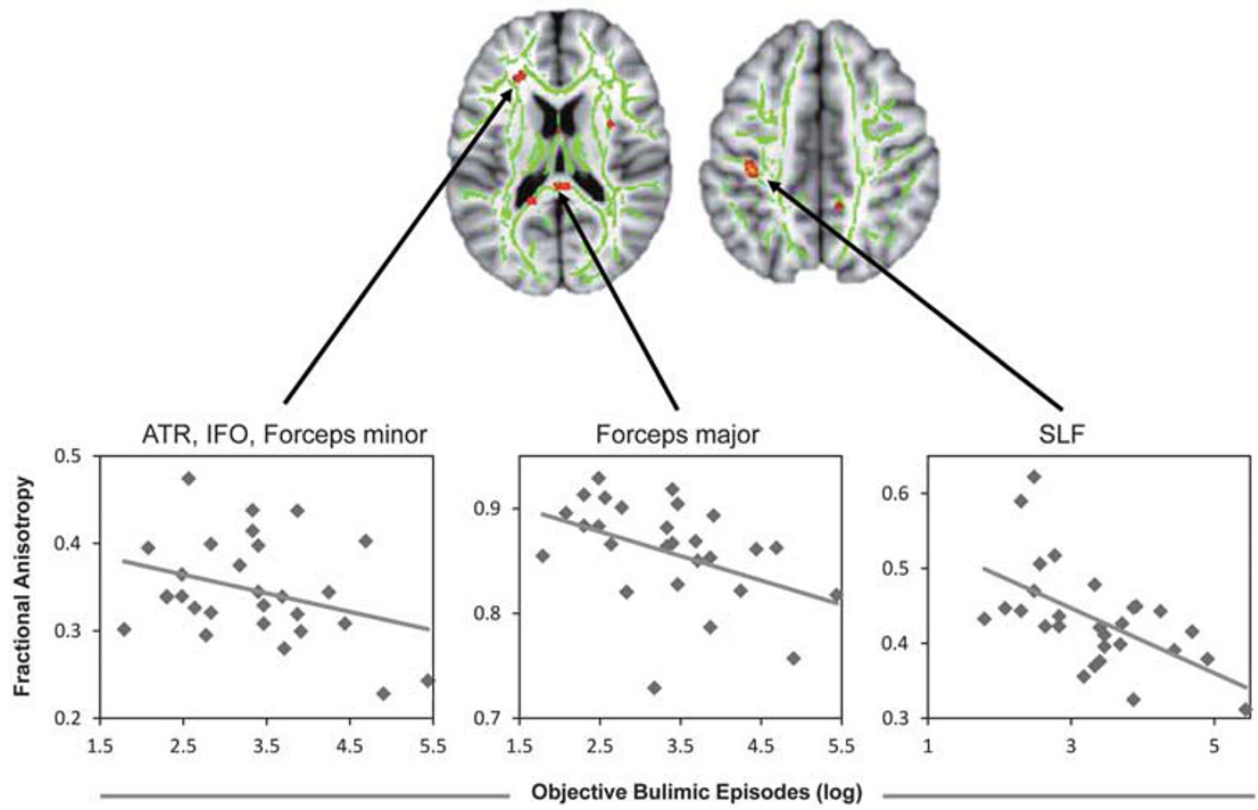

Figure 2 Correlations of FA with objective bulimic episodes in the BN group. Significant correlations of FA with objective bulimic episodes within the past 28 days before the MRI scan were detected in forceps minor and major, left ATR, IFO, and SLF. Logarithmic transformation was used to reduce excessive skewness in this frequency variable. FA values, adjusted for age and duration of illness, are plotted on the y axis, and log-transformed objective bulimic episodes are plotted on the $x$ axis. The scatter shows less FA in those with more severe symptoms. These results are 'thickened' using tbss_fill script in FSL TBSS toolbox and displayed in neurological orientation.

\section{DISCUSSION}

Significantly less FA was detected in BN compared with healthy individuals in WM tracts extending through bilateral frontal and temporoparietal areas, including forceps minor and major, SLF, SLF-temporal, IFO, ATR, CST, UNC, and cingulum. In the $\mathrm{BN}$ group, greater $\mathrm{FA}$ reductions in forceps, left ATR, IFO, and SLF were detected in those with the most severe symptoms, and increased $\mathrm{RD}$ was detected in the same left hemisphere tracts where FA was reduced compared with healthy participants. Significant diagnosis-by-age interactions on FA and RD in forceps and left ATR, CST, and SLF were driven by the $\mathrm{BN}$ group in which $\mathrm{FA}$ increased and $\mathrm{RD}$ decreased with advancing age in these tracts. Moreover, group differences in FA and RD were more widespread in adolescent participants, suggesting that abnormalities in WM microstructure arise early in the course of BN. Finally, significant diagnosis-by-Stroop interactions in forceps minor, right ATR and IFO, left CST, and SLF indicated that greater Stroop interference accompanied greater FA reductions in the $\mathrm{BN}$ group, suggesting that microstructural abnormalities in these tracts may be associated with functional deficits in self-regulation in BN.

Our previous fMRI findings from adult (Marsh et al, 2009) and adolescent (Marsh et al, 2011) females with BN indicated deficient activation of frontal areas compared with age-matched control participants during performance of a self-regulatory task. Our structural findings suggested smaller local volumes of left inferior frontal and bilateral temporoparietal regions in the same $\mathrm{BN}$ compared with healthy participants (Marsh et al, 2015). In addition, reductions in inferior frontal cortices were associated with more severe symptoms, advancing age, and greater Stroop interference in the $\mathrm{BN}$ group. The current findings of reduced FA in SLF, IFO, ATR, CST, UNC, and cingulum suggest that alterations in the microstructure of WM pathways extending to and from frontal and temporoparietal cortices are also present in adolescents with $\mathrm{BN}$.

Forceps minor is the frontal portion of the corpus callosum. Thus, our finding of reduced FA in forceps minor is consistent with previous findings of reduced FA in the corpus callosum in adults with BN compared with healthy women (Mettler et al, 2013). These data were interpreted in light of human and nonhuman data implicating the corpus callosum in taste processing (Fabri and Polonara, 2013). Reduced FA in the corpus callosum has also been reported in adolescent binge drinkers (McQueeny et al, 2009) and is associated with increased impulsivity in cocaine dependence (Moeller et al, 2005), suggesting a common potential mechanism for binge-type and impulsive behaviors across eating and substance disorders. In contrast, reduced FA in the CST may be specific to BN and interpreted in terms of the physiological effects of binge eating and purging. The CST is a descending pathway that connects the cerebral cortex with the spinal cord and is involved in voluntary or skilled movements. Nerves within the CST support movement in the muscles of the head that are involved in swallowing, phonation, and movements of the tongue (Young et al, 2008). As such, microstructural abnormalities within the CST may perhaps be a product of the illness, given the detrimental effects of binge eating and purging on oropharyngeal swallow patterns in BN (Roberts et al, 1989).

We also detected less FA in the IFO and SLF in BN compared with healthy participants. These are major 


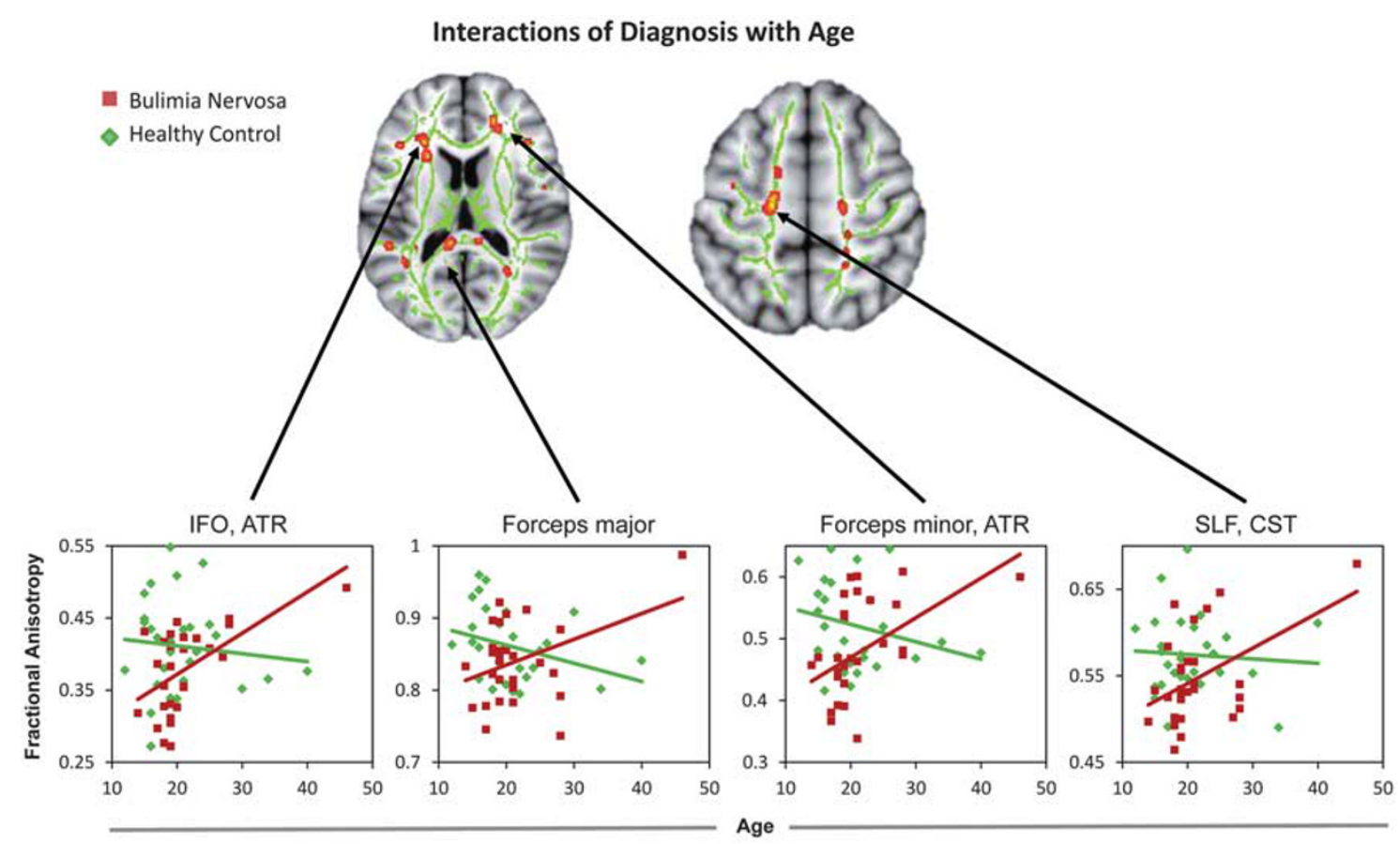

Figure 3 Correlations of FA with age in the BN versus control groups. FA in forceps minor and major, bilateral ATR, CST, IFO, and left SLF correlated positively with age in the BN participants but not the healthy participants, producing significant diagnosis $\times$ age interactions. FA is plotted on the $y$ axis and age is plotted on the $x$ axis. The results are 'thickened' using tbss_fill script in FSL TBSS toolbox and displayed in neurological orientation.

association pathways that connect frontal cortices with temporal and occipital lobes (IFO) (Kier et al, 2004) and with parietal areas (SLF I, II, III) and superior temporal gyrus (Makris et al, 2005). Both regional volume (Takeuchi et al, 2013) and integrity (Peters et al, 2014) of the IFO are associated with performance on tasks of executive functions in healthy individuals, regardless of age. These findings particularly implicate the IFO in visual processing, consistent with its frontal connections to occipital and temporal visual association cortices (Martino et al, 2010). Less FA in this tract has also been reported in women with AN compared with healthy women (Kazlouski et al, 2011), data suggested to relate to body image disturbances that may be due, in part, to poor communication across occipital, temporal, and frontal cortices. Findings from both animals and humans suggest that body image perceptions may be represented within the parietal cortex (Gaudio et al, 2014; Iriki et al, 2001). Given that three of the SLF components connect parietal and frontal regions, microstructural alterations in these WM fibers might also contribute to the impaired regulation of body representations in eating disorders. Although we did not have a direct measure of body image, FA reductions in IFO and SLF were greatest in the BN participants who were most preoccupied with their shape and weight. Thus, although speculative, reduced FA in these tracts may be associated with the impaired processing and regulation of body image in individuals with eating disorders, consistent with findings of reduced FA in individuals with AN (Kazlouski et al, 2011; Via et al, 2014) and body dysmorphic disorder (Buchanan et al, 2013) compared with healthy participants. Reduced FA in IFO and SLF is also consistent with our previous findings of smaller local volumes of bilateral temporoparietal areas in BN compared with control participants (Marsh et al, 2015), suggesting abnormalities in both gray and WM in these brain areas (Joos et al, 2010).

Our findings of less FA in the UNC, cingulum, and ATR may relate to the previously documented fMRI findings of reward dysfunction in individuals both ill and recovered from BN (Frank et al, 2013). The UNC and cingulum are major limbic tracts (Catani and Thiebaut de Schotten, 2008), whereas the ATR connects thalamus to medial prefrontal cortex, with further connections to medial temporal regions (ie, hippocampus and amygdala) that are involved in reward processing (Haber and Knutson, 2010). The integrity of these tracts may influence reward processing functions, as FA in these tracts is associated with reward-related BOLD response in the nucleus accumbens in healthy individuals (Camara et al, 2010; Koch et al, 2014). As the ATR is hypothesized to be part of an emotional/motivational system and relevant for the regulation of mood (Coenen et al, 2012), less FA in this tract may also relate to mood dysregulation in individuals with $\mathrm{BN}$.

The only extant DTI study of individuals with BN (Mettler et al, 2013) used a different methodology for data analysis (SPM $v s$ TBSS) and a different atlas for anatomical labeling (Orrison, 1995 versus Oishi et al, 2011). Nevertheless, our findings converge in showing that FA is reduced in $\mathrm{BN}$ in multiple WM tracts. Unlike the prior study, we also assessed age effects on group differences in WM microstructure and observed diagnosis-by-age interactions on FA in tracts where group differences were detected (ie, forceps minor and major, ATR, CST, IFO, and left SLF), deriving from significant positive associations with age in the BN group. Together with our finding that FA reductions were more widespread in the BN compared with healthy adolescents, these data suggest that WM microstructural abnormalities may arise early in BN but may not contribute to illness persistence, as FA reductions were not associated with illness 
duration (Supplementary Material). Developmental DTI data from healthy individuals suggest that age-related increases in FA are primarily driven by reductions in RD (Giorgio et al, 2008), a measure that rodent post-mortem data suggest may reflect the degree of myelination (Song et al, 2002). The interpretation of changes in $\mathrm{RD}$ over development is less clear in humans. For example, RD reductions and FA increases in a given tract could also result from more coherent alignment of fibers or fewer crossing fibers (Jones et al, 2013). Nevertheless, our finding that RD was inversely associated with age in multiple left hemisphere tracts in the BN group may suggest an abnormal trajectory of WM development in BN. Future longitudinal studies should test this hypothesis directly, given that protracted myelination seems to underlie many different disease phenotypes (Haroutunian et al, 2014).

Although FA in multiple tracts increased with advancing age in the $\mathrm{BN}$ group, less FA in some of these tracts (forceps minor, right ATR and IFO, left CST, and SLF) was associated with greater Stroop interference in BN. As significant group differences in FA and $\mathrm{RD}$ were not detected in adults (when analyzed separately), the effects of Stroop performance and symptom severity could not be tested in the adult subsample because these exploratory analyses entailed using FA (or RD) values extracted from clusters on maps depicting significant group differences. Thus, the adolescents in our BN sample likely contributed to the association of less FA with greater Stroop interference in that group. Stroop interference typically decreases with advancing age in healthy individuals (Marsh et al, 2006), likely reflecting the functional maturation of frontostriatal and frontoparietal attentional control circuits. Integrity of the SLF is associated with the ability to orient attention on another cognitive task in healthy adolescents (Ge et al, 2013), and less FA in the IFO is reported in adults with ADHD (Shaw et al, 2015). Thus, less FA in these tracts in adolescents with BN may contribute to their difficulty in mobilizing the attentional resources necessary to avoid word reading on the Stroop task and to avoid the cues that trigger their binge-eating episodes in daily life.

This study is limited by its modest sample size, crosssectional design, and the heterogeneity of our BN sample that included participants at differing stages of treatment and who varied in symptom severity. In addition, our BN and control groups differed in verbal IQ, but this difference was driven by the adults in our sample (Supplementary Table S1) and therefore unlikely driving the group differences in FA that remained significant in the adolescents when tested separately. Although we did not account for menstrual status, which could affect neural functioning in women (Dreher et al, 2007), we do not suspect that FA would be affected, nor do we suspect that menstrual status differed systematically enough across groups to confound our findings. Another limitation was our use of acquisition parameters that recent advances have shown to be suboptimal. For example, the acquisition of at least 30 gradient directions and isotropic voxels is now recommended (Jones et al, 2013), and newer parameters may reduce the crossing fiber issue (Jbabdi et al, 2010). Finally, our use of the Stroop task to assess self-regulatory control in this study was not ideal, given that Stroop interference was not associated with BN symptoms in our sample.
Future studies of individuals with BN should employ tractography to further probe the WM microstructural abnormalities uncovered herein and enhance our understanding of anatomical connectivity in BN. Such future studies should continue probing associations of anatomical connections with brain function and behavior in BN. Future longitudinal studies comparing brain development in $\mathrm{BN}$ and healthy adolescents, beginning at illness onset, are required to determine whether our cross-sectional findings of age effects on WM microstructure in the BN group reflect abnormal maturation or neurodevelopmental abnormalities in $\mathrm{BN}$ or, instead, the effects of chronic illness on WM microstructure.

\section{FUNDING AND DISCLOSURE}

The authors declare no conflict of interest.

\section{ACKNOWLEDGMENTS}

This work was supported in part by NIMH grants K01MH077652 and R01MH090062 (to RM), by grants from the National Alliance for Research on Schizophrenia and Depression (NARSAD, to RM and $\mathrm{XH}$ ), and by funding from the Sackler Institute for Developmental Psychobiology, Columbia University.

\section{REFERENCES}

Asato MR, Terwilliger R, Woo J, Luna B (2010). White matter development in adolescence: a DTI study. Cereb Cortex 20: 2122-2131.

Buchanan BG, Rossell SL, Maller JJ, Toh WL, Brennan S, Castle DJ (2013). Brain connectivity in body dysmorphic disorder compared with controls: a diffusion tensor imaging study. Psychol Med 43: 2513-2521.

Camara E, Rodriguez-Fornells A, Munte TF (2010). Microstructural brain differences predict functional hemodynamic responses in a reward processing task. J Neurosci 30: 11398-11402.

Catani M, Thiebaut de Schotten M (2008). A diffusion tensor imaging tractography atlas for virtual in vivo dissections. Cortex 44: 1105-1132.

Coenen VA, Panksepp J, Hurwitz TA, Urbach H, Madler B (2012). Human medial forebrain bundle (MFB) and anterior thalamic radiation (ATR): imaging of two major subcortical pathways and the dynamic balance of opposite affects in understanding depression. J Neuropsychiatry Clin Neurosci 24: 223-236.

Dreher JC, Schmidt PJ, Kohn P, Furman D, Rubinow D, Berman KF (2007). Menstrual cycle phase modulates reward-related neural function in women. Proc Natl Acad Sci USA 104: 2465-2470.

Fabri M, Polonara G (2013). Functional topography of human corpus callosum: an FMRI mapping study. Neural Plast 2013: 251308.

Frank GK, Shott ME, Hagman JO, Mittal VA (2013). Alterations in brain structures related to taste reward circuitry in ill and recovered anorexia nervosa and in bulimia nervosa. Am J Psychiatry 170: 1152-1160.

Gaudio S, Brooks SJ, Riva G (2014). Nonvisual multisensory impairment of body perception in anorexia nervosa: a systematic review of neuropsychological studies. PLoS One 9: e110087.

Ge H, Yin X, Xu J, Tang Y, Han Y, Xu W et al (2013). Fiber pathways of attention subnetworks revealed with tract-based spatial statistics (TBSS) and probabilistic tractography. PLoS One 8: e78831. 
Giedd JN, Rapoport JL (2010). Structural MRI of pediatric brain development: what have we learned and where are we going? Neuron 67: 728-734.

Giorgio A, Watkins KE, Douaud G, James AC, James S, De Stefano $\mathrm{N}$ et al (2008). Changes in white matter microstructure during adolescence. Neuroimage 39: 52-61.

Haber SN, Knutson B (2010). The reward circuit: linking primate anatomy and human imaging. Neuropsychopharmacology 35: 4-26.

Haroutunian V, Katsel P, Roussos P, Davis KL, Altshuler LL, Bartzokis G (2014). Myelination, oligodendrocytes, and serious mental illness. Glia 62: 1856-1877.

He XF, Liu W, Li XZ, Li QL, Liu F, Rauh VA et al (2014). Automated assessment of the quality of diffusion tensor imaging data using color cast of color-encoded fractional anisotropy images. Magn Reson Imaging 32: 446-456.

Iriki A, Tanaka M, Obayashi S, Iwamura Y (2001). Self-images in the video monitor coded by monkey intraparietal neurons. Neurosci Res 40: 163-173.

Jbabdi S, Behrens TE, Smith SM (2010). Crossing fibres in tractbased spatial statistics. Neuroimage 49: 249-256.

Jones DK, Knosche TR, Turner R (2013). White matter integrity, fiber count, and other fallacies: the do's and don'ts of diffusion MRI. Neuroimage 73: 239-254.

Joos A, Kloppel S, Hartmann A, Glauche V, Tuscher O, Perlov E et al (2010). Voxel-based morphometry in eating disorders: correlation of psychopathology with grey matter volume. Psychiatry Res 182: 146-151.

Kazlouski D, Rollin MD, Tregellas J, Shott ME, Jappe LM, Hagman JO et al (2011). Altered fimbria-fornix white matter integrity in anorexia nervosa predicts harm avoidance. Psychiatry Res 192: 109-116.

Kier EL, Staib LH, Davis LM, Bronen RA (2004). MR imaging of the temporal stem: anatomic dissection tractography of the uncinate fasciculus, inferior occipitofrontal fasciculus, and Meyer's loop of the optic radiation. AJNR Am J Neuroradiol 25: 677-691.

Koch K, Wagner G, Schachtzabel C, Schultz CC, Gullmar D, Reichenbach JR et al (2014). Association between white matter fiber structure and reward-related reactivity of the ventral striatum. Hum Brain Mapp 35: 1469-1476.

Lebel C, Beaulieu C (2011). Longitudinal development of human brain wiring continues from childhood into adulthood. J Neurosci 31: 10937-10947.

Lebel C, Walker L, Leemans A, Phillips L, Beaulieu C (2008). Microstructural maturation of the human brain from childhood to adulthood. Neuroimage 40: 1044-1055.

Makris N, Kennedy DN, McInerney S, Sorensen AG, Wang R, Caviness VS Jr et al (2005). Segmentation of subcomponents within the superior longitudinal fascicle in humans: a quantitative, in vivo, DT-MRI study. Cereb Cortex 15: 854-869.

Marsh R, Horga G, Wang Z, Wang P, Klahr KW, Berner LA et al (2011). An FMRI study of self-regulatory control and conflict resolution in adolescents with bulimia nervosa. Am J Psychiatry 168: $1210-1220$.

Marsh R, Stefan M, Bansal R, Hao X, Walsh BT, Peterson BS (2015). Anatomical characteristics of the cerebral surface in bulimia nervosa. Biol Psychiatry 77: 616-623.

Marsh R, Steinglass JE, Gerber AJ, Graziano O'Leary K, Wang Z, Murphy D et al (2009). Deficient activity in the neural systems that mediate self-regulatory control in bulimia nervosa. Arch Gen Psychiatry 66: 51-63.

Marsh R, Zhu H, Schultz RT, Quackenbush G, Royal J, Skudlarski P et al (2006). A developmental fMRI study of self-regulatory control. Hum Brain Mapp 27: 848-863.

Martino J, Brogna C, Robles SG, Vergani F, Duffau H (2010). Anatomic dissection of the inferior fronto-occipital fasciculus revisited in the lights of brain stimulation data. Cortex 46: 691-699.

McQueeny T, Schweinsburg BC, Schweinsburg AD, Jacobus J, Bava S, Frank LR et al (2009). Altered white matter integrity in adolescent binge drinkers. Alcohol Clin Exp Res 33: 1278-1285.

Mettler LN, Shott ME, Pryor T, Yang TT, Frank GK (2013). White matter integrity is reduced in bulimia nervosa. Int J Eat Disord 46: 264-273.

Moeller FG, Hasan KM, Steinberg JL, Kramer LA, Dougherty DM, Santos RM et al (2005). Reduced anterior corpus callosum white matter integrity is related to increased impulsivity and reduced discriminability in cocaine-dependent subjects: diffusion tensor imaging. Neuropsychopharmacology 30: 610-617.

Nichols TE, Holmes AP (2002). Nonparametric permutation tests for functional neuroimaging: a primer with examples. Hum Brain Mapp 15: 1-25.

Oishi K, Faria A, van Zijl PC, Mori S(eds) (2011). MRI Atlas of Human White Matter. Academic Press.

Orrison WW (1995). Atlas of Brain Function. Thieme Medical Publishers; Georg Thieme Verlag: New York Stuttgart. viiipp 176.

Peters BD, Ikuta T, DeRosse P, John M, Burdick KE, Gruner P et al (2014). Age-related differences in white matter tract microstructure are associated with cognitive performance from childhood to adulthood. Biol Psychiatry 75: 248-256.

Roberts MW, Tylenda CA, Sonies BC, Elin RJ (1989). Dysphagia in bulimia nervosa. Dysphagia 4: 106-111.

Romer D (2010). Adolescent risk taking, impulsivity, and brain development: implications for prevention. Deve Psychobiol 52: 263-276.

Shaw P, Sudre G, Wharton A, Weingart D, Sharp W, Sarlls J (2015). White matter microstructure and the variable adult outcome of childhood attention deficit hyperactivity disorder. Neuropsychopharmacology 40: 746-754.

Smith SM (2002). Fast robust automated brain extraction. Hum Brain Mapp 17: 143-155.

Smith SM, Jenkinson M, Johansen-Berg H, Rueckert D, Nichols TE, Mackay CE et al (2006). Tract-based spatial statistics: voxelwise analysis of multi-subject diffusion data. Neuroimage 31: 1487-1505. Smith SM, Jenkinson M, Woolrich MW, Beckmann CF, Behrens TE, Johansen-Berg $\mathrm{H}$ et al (2004). Advances in functional and structural MR image analysis and implementation as FSL. Neuroimage 23: S208-S219.

Smith SM, Nichols TE (2009). Threshold-free cluster enhancement: addressing problems of smoothing, threshold dependence and localisation in cluster inference. Neuroimage 44: 83-98.

Song SK, Sun SW, Ramsbottom MJ, Chang C, Russell J, Cross AH (2002). Dysmyelination revealed through MRI as increased radial (but unchanged axial) diffusion of water. Neuroimage 17: 1429-1436.

Stroop JR (1935). Studies of interference in serial verbal reactions. J Exp Psychology 18: 643-662.

Takeuchi H, Taki Y, Sassa Y, Hashizume H, Sekiguchi A, Fukushima A et al (2013). Brain structures associated with executive functions during everyday events in a non-clinical sample. Brain Struct Funct 218: 1017-1032.

Via E, Zalesky A, Sanchez I, Forcano L, Harrison BJ, Pujol J et al (2014). Disruption of brain white matter microstructure in women with anorexia nervosa. J Psychiatry Neurosci 39: 130135.

Walsh BT, Wilson GT, Loeb KL, Devlin MJ, Pike KM, Roose SP et al (1997). Medication and psychotherapy in the treatment of bulimia nervosa. Am J Psychiatry 154: 523-531.

Young PA, Young PH, Tolbert DL, Young PA (2008). Basic Clinical Neuroscience2nd ednWolters Kluwer Health/Lippincott Williams \& Wilkins: Philadelphia. pp 69-70.

Supplementary Information accompanies the paper on the Neuropsychopharmacology website (http://www.nature.com/npp) 